\title{
Fußmatte hilft, Rezidivulkus-Risiko zu senken
}

Fragestellung: Zur Evaluation einer neuartigen Fußmatte zur automatischen Temperaturmessung an der plantaren Fußsohle wurde eine Kohorte von Diabetikern mit abgeheilten plantaren Ulzera herangezogen. Es wurde untersucht, ob Temperaturdifferenzen, gemessen mit dieser Fußmatte, das Auftreten von Rezidivulzera vorhersagen können.

Hintergrund: Patienten mit Zustand nach Abheilung von plantaren neuropathischen Ulzera haben ein extrem hohes Risiko, ein Rezidivulkus zu entwickeln. Mehrere Studien wiesen eine Rezidivrate von 30 bis $40 \%$ in einem Jahr nach Abheilung nach, trotz guter Versorgung mit Schuhwerk und Podologie. Eine Vermeidung dieser Rezidive wäre von außerordentlichem Nutzen für die Patienten.

Es konnte gezeigt werden, dass die regelmäßige Inspektion der Füße bei der Prophylaxe von Rezidivulzera nur wenig erfolgreich ist. In drei randomisierten Studien konnte hingegen durch Kontrolle der Temperatur der Füße eine Reduktion der Rezidivulzera von bis zu $70 \%$ nachgewiesen werden. Hierbei wird die Temperatur des Fußes mit der Gegenseite verglichen, wobei eine Differenz von mehr als 2,2 Grad Celsius (4 Grad Fahrenheit) als suspekt angesehen wird. Obwohl diese sehr erfolgversprechenden Studien bereits 2007 und früher publiziert wurden, hat sich die Temperaturmessung der Füße zur Rezidivprophylaxe von Ulzera bisher nicht durchgesetzt.

Patienten und Methoden: 132 Patienten mit Diabetes und abgeheiltem plantarem neuropathischem Ulkus ohne hochgradige pAVK wurden in diese multizentrische, prospektive Kohortenstudie aufgenommen. Den Patienten wurde eine Fußmatte (Remote Temperature Monitoring System; Podimetrics, Inc., Somerville, MA) mit nach Hause gegeben, die beim Betreten automatisch die Temperatur beider Fußsohlen registriert und die Ergebnisse direkt telemetrisch versendet. Die Fußmatte kann ohne Installation von den Patienten direkt genutzt werden. Sie besteht in der Oberfläche aus einem verformbaren Schaummaterial, das einen direkten Kontakt der gesamten Fußsohle mit 2.000 Temperatursensoren erlaubt.

Nach ruhigem Stehen von 20 Sekunden Dauer wird dem Patienten angezeigt, dass die Messung beendet ist und gleichzeitig erfolgt die automatische Übersendung der Befunde ans Fußzentrum. In der Auswertung der Ergebnisse wurden die beiden Kohorten mit und ohne Rezidivulkus verglichen. Außerdem erfolgte eine Untersuchung dahingehend, welche Temperaturdifferenz die höchste Sensitivität und Spezifität bezüglich

\section{Originalie}

Fryberg RG, Gordon IL, Reyzelman AM et al. Feasibility and Efficacy of a Smart Mat Technology to Predict Development of Diabetic Plantar Ulcers.

Diabetes Care 2017;40:973-80 der Vorhersage eines drohenden Rezidivulkus hat. Der Zeitraum der Untersuchung betrug 34 Wochen.

Ergebnisse: Von den $129 \mathrm{~Pa}$ tienten mit auswertbaren Daten hatten 37 (29\%) ein oder mehrere Rezidivulzera. Insgesamt traten 53 Rezidivulzera auf, entsprechend 0,62 Ulzera pro Patient und Jahr. Bei einer Temperaturdifferenz von 2,2 Grad Celsius identifizierte das System 97 \% der Ulzera, mit einer durchschnittlichen Latenz von 37 Tagen. Bei dieser Temperaturdifferenz kam es bei $57 \%$ zu einem falsch positiven Ergebnis. Bei einer Temperaturdifferenz von 3,2 Grad Celsius betrug die Sensitivität $70 \%$ und die Spezifität $32 \%$. Ungefähr 86 \% der Teilnehmer nutzten die Fußmatte mindestens 3 Tage in der Woche.

Schlussfolgerungen: Die Autoren sehen einen erheblichen Fortschritt in der Prophylaxe von Rezidivulzera durch diese Temperatur-Fußmatte. Da der Anstieg der Fußtemperatur bereits im Mittel 5 Wochen vor dem Auftreten des Ulkus erfolgt, bleibt genügend Zeit, um Prophylaxe zu betreiben, insbesondere am Schuhwerk. Andererseits kommt es bei einer Temperaturdifferenz von 2,2 Grad Celsius, bei Berücksichtigung auch der falsch positiven Messungen, nur zu ca. drei Vorstellungen pro Patient und Jahr in diesem Kollektiv. Diese automatische Fußmatte wird aufgrund der Einfachheit der Durchführung der Messungen positiv von den Patienten angenommen, was eine gute Compliance zur Folge hat.

\section{- Kommentar von Prof. Dr. med. M. Spraul}

\section{Das sollte Kassenleistung werden}

Die Messung der Temperatur der Fußsohle ermöglicht, entsprechend den Interventionsstudien aus den Jahren 2007 und 2004, nicht nur die Vorhersage von Rezidivulzera, sondern auch eine signifikante Reduktion des Auftretens von Ulzera überhaupt. Dennoch kam diese Methode seither kaum zur Anwendung. Dies lag vermutlich an den Schwierigkeiten, regelmäßig die Temperatur des Fußes zu erfassen. Die vorliegende Studie mit einer automatisch messenden und -sendenden Temperatur-Fußmatte erbrachte wichtige neue Daten zur Genauigkeit und Anwendbarkeit dieser Methode. Aus meiner Sicht ist dies eine außerordentlich wichtige Methode zur Reduzierung der erheblichen Morbidität durch Ulzera bei Hochrisikopatienten. Eine Erstattung der Kosten durch die Krankenkassen sollte für ein solch wichtiges Selbstmonitoring in nicht zu ferner Zukunft möglich sein.

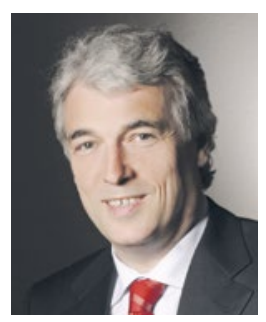

Prof. Dr. med. Maximilian Spraul

Mathias-Spital \& Jakobi-Krankenhaus Interdisziplinäres Diabetes-Fuß-Zentrum Frankenburgstr. 31, 48431 Rheine m.spraul@mathias-spital.de 